\title{
Effects of Combination of Anti-CTLA-4 and Anti-PD-1 on Gastric Cancer Cells Proliferation, Apoptosis and Metastasis
}

\author{
Bin Wang ${ }^{\mathrm{a}} \quad$ Lei Qin $^{\mathrm{a}} \quad$ Mei Ren ${ }^{\mathrm{b}}$ Hao Sun ${ }^{\mathrm{a}}$ \\ aDepartment of Gastrointestinal Surgery, Jining No.1 People's Hospital, Jining, ${ }^{\mathrm{b} D e p a r t m e n t}$ of \\ Oncology, Jining No.1 People's Hospital, Jining, China
}

\section{Key Words}

Gastric cancer $•$ Anti-CTLA-4 antibody $•$ Anti-PD-1 antibody $•$ EMT $\bullet \beta$-catenin

\begin{abstract}
Background/Aims: Gastric cancer (GC) is one of the most common and lethal varieties of cancers. Anticancer activities of anti-CTLA-4 and anti-PD-1 antibodies have been explored in different cancers, including GC. The study aimed to explore the role of combination therapy with anti-CTLA-4 and anti-PD-1 antibodies in GC cells, and understand the possible underlying molecular mechanism. Methods: MKN-45 and MGC-803 cells were divided into four groups, namely control, CTLA-4, PD-1, and CTLA-4\&PD-1. Cell viability, cell cycle, apoptosis, migration and invasion were measured by MTT, flow cytometry, and transwell assays, respectively. Expression levels of different mRNAs and proteins associated with apoptosis, epithelial mesenchymal transition (EMT), $\beta$-catenin, MAPK, and PI3K/AKT pathways were assessed by RT-qPCR and western blot analysis, respectively. The tumor formation in vivo was examined by tumor Xenograft model assay. Results: Combination with anti-CTLA-4 and anti-PD-1 antibodies significantly suppressed cell proliferation, induced apoptosis, as well as inhibited migration, invasion, and EMT in MKN-45 and MGC-803 cells. Western blotting revealed that combination with anti-CTLA-4 and anti-PD- 1 antibodies declined the activation of $\beta$-catenin, MAPK and PI3K/AKT signal pathways. Moreover, combination of anti-CTLA-4 and anti-PD-1 antibodies inhibited tumor formation in vivo. Furthermore, the mRNA levels of CTLA-4 and PD-1 were significantly decreased in si-CTLA and si-PD-1 transfected cells, and combination with si-CTLA and si-PD-1 also suppressed cell proliferation, migration, invasion, EMT and induced apoptosis in MKN-45 cells. Conclusion: Combination therapy with antiCTLA-4 and anti-PD-1 antibodies presented the promising outcomes in GC, although further investigations are warranted.
\end{abstract}

\begin{tabular}{ll}
\hline Hao Sun & Department of Gastrointestinal Surgery, Jining No.1 People's Hospital, \\
& No.6, Jiankang Road, Jining 272011 (China) \\
& E-Mail sunh080@sina.com
\end{tabular}




\section{Introduction}

Gastric cancer (GC) is one of the most common cancers, and is the fifth most common type worldwide [1]. It is the second most common cause of cancer-related deaths after lung cancer [2, 3]. In 2013, about 984, 000 new cases of GC were diagnosed, and about 841, 000 deaths were documented due to GC [4]. Treatment options for GC are decided based on the stage and type of cancer, and the different treatment modalities include surgical removal of malignancy or via endoscopic resection, surgery, chemotherapy, and radiotherapy [58]. Despite recent advances in the treatment strategies for GC, the prognosis of GC is still grave [8]. Currently, immunotherapy has become one of the important treatment modalities for management of different varieties of cancers including GC $[1,9,10]$. Studies have already established that host immunity plays a major role in defensing the occurrence and development of tumors $[1,11]$. Cancerous cells are known to evade the host immune surveillance, thereby facilitating its invasive capability [12-16].

It has been established that an increase in natural regulatory $\mathrm{T}$ lymphocytes (Treg) is closely associated with tumor progression in many cancer patients [16]. Cytotoxic T-lymphocyte-associated antigen 4 (CTLA-4) is one of the major regulatory factors for activating T-cells. Constitutively, T-cells are expressed on cell surface of Treg and its expression can be activated T lymphocytes and monocytes [12]. Its structure is similar with costimulatory molecules, such as CD28, as well as it has high affinity to the same receptor (CD80/86) [12]. It is well known that up-regulation of CTLA-4 can reduce the expression levels of interleukin-2 (IL-2) and its receptor. Moreover, CTLA-4 can arrest T cells at G1 phase. Several studies have explored the outcomes of CTLA-4 blockade with corresponding monoclonal antibodies in different types of cancers $[12,15,16]$.

Programmed cell death protein 1 (PD-1) is a type of immunoglobulin, which is mainly expressed on the surface of activated CD4+/CD8+ T cells, B lymphocytes, natural killer (NK) cells, and myeloid cells [17]. Recent study demonstrated that PD-1 expression could suppress the activation of $\mathrm{T}$ cells, thereby playing an important role in immune tolerance. Moreover, PD-1 suppressed the action of T cells through binding to programmed death-1 ligand1 (PDL1) and programmed death-1 ligand2 (PD-L2) [17]. Increasing evidences have established that the action of PD-1 contributes to the tumor cells to evade immune surveillance, thereby improving the invasive capability of the tumor cells [5, 17-19]. Furthermore, several studies have explored the anticancer potential of antibodies against PD-1 [17-20]. However, the effect of CTLA-4 and PD-1 on GC cells remains unclear.

Hence, the present study aimed to explore the anticancer effect of combination of antiCTLA-4 and anti-PD-1 antibodies in GC cells, as well as to uncover its possible underlying molecular mechanism.

\section{Materials and Methods}

\section{Cell Culture and Treatment}

MKN-45 cells with high metastatic potential, MGC-803 cells with low metastatic potential used in the present study experiments were obtained from The Second Hospital of Jilin University (Jilin, China). The cells were maintained in Dulbecco's modified Eagle's medium (DMEM, Gibco BRL, Rockville, MD, USA) with $10 \%$ fetal bovine serum (FBS, Life Technologies, Carlsbad, CA, USA), $100 \mathrm{U} / \mathrm{mL}$ penicillin at $37^{\circ} \mathrm{C}$ in a humidified atmosphere containing $5 \% \mathrm{CO}_{2}$.

Antibodies of anti-CTLA-4 (ab210381, 1:1000) and anti-PD-1 (ab140950, 1: 1000, Abcam, Cambridge, UK) were used to treat these cells. MKN-45 and MGC-803 cell lines were divided into four groups, namely as control group (cells were not treated with either of the two antibodies, CTLA-4 or PD-1 antibodies), CTLA-4 treated group (cells were treated with anti-CTLA-4 antibodies), PD-1 treated group (cells were treated with anti-PD-1 antibodies) and CTLA-4\&PD-1 group (receiving combined therapy with anti-CTLA-4 and antiPD-1 antibodies).

\section{KARGER}




\section{Cellular Physiology Cell Physiol Biochem 2018;49:260-270 \begin{tabular}{l|l|l} 
DOI: 10.1159/000492876 & $\begin{array}{l}\text { (O) 2018 The Author(s). Published by S. Karger AG, Basel } \\
\text { www.karger.com/cpb }\end{array}$
\end{tabular}}

Wang et al.: Effects of Anti-CTLA-4 and Anti-PD-1 on GC

\section{Cell Transfection}

The CTLA-4-specific and PD-1-specific small interference RNA (siRNA) were constructed by GenPharma (Shanghai, China) to inhibit the RNA levels of CTLA-4 and PD-1. All transfections were detected by using the Lipofectamine 2000 reagent (Invitrogen, CA, USA) according to the manufacturer's protocol.

\section{Cell Viability Assay}

The MKN-45 and MGC-803 cells were seeded in 96-well plates at a density of $3 \times 10^{3} /$ well. After incubation for $24 \mathrm{~h}, 48 \mathrm{~h}$ and $72 \mathrm{~h}, 10 \mu \mathrm{L}$ Cell Counting Kit-8 (CCK-8, Dojin Laboratories, Kumamoto, Japan) was added to the culture plates, and the plates were incubated for $1 \mathrm{~h}$ at $37^{\circ} \mathrm{C}$ in humidified $95 \%$ air and $5 \% \mathrm{CO}_{2}$. The absorbance was measured at $450 \mathrm{~nm}$ using a Microplate Reader (Bio-Rad, Hercules, CA, USA).

\section{Cell Cycle Assay}

Cell cycle analysis was performed by using the Cell Cycle and Apoptosis Analysis Kit (Beyotime, Shanghai, China) according to the manufacturer's instructions. After treatment, these cells were washed twice with phosphate-buffered saline (PBS) and fixed in $70 \%$ ethanol at $4^{\circ} \mathrm{C}$ overnight. Afterward, these

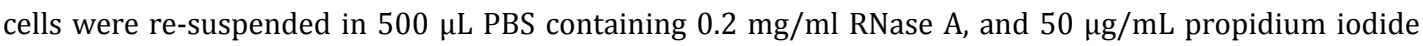
(PI) was added to stain these cells for $30 \mathrm{~min}$ in the dark at room temperature. The percentages of cells occupying the different phases (G0/G1, S, and G2/M) of the cell cycle were counted and compared using FACScan flow cytometer (Becton Dickinson, San Jose, USA).

\section{Migration and Invasion Assay}

Cell migration and invasion were assessed by transwell chamber with 8.0- $\mu \mathrm{M}$ pore size (Corning, Cambridge, MA, USA). Briefly, $200 \mu \mathrm{L}$ MKN-45 and MGC-803 cells suspensions were added into the upper chamber, and $600 \mu \mathrm{L}$ of complete medium was then added to the lower chamber of each well. After incubation for $48 \mathrm{~h}$, these cells were stained with Giemsa (Sigma, St. Louis, MO, USA) for $30 \mathrm{~min}$. Non-traversed cells were removed by a wet cotton swab. The cell invasion assay was carried out similarly, except that $0.8 \mathrm{mg} /$ $\mathrm{mL}$ (Matrigel BD Biosciences) was added to each well to incubate for $6 \mathrm{~h}$ before cells were seeded on the membrane.

\section{Apoptosis assay}

Cells were harvested using $0.25 \%$ trypsin-EDTA and were washed twice in ice-cold PBS. Then, cells were suspended in $100 \mu \mathrm{L} 1 \times$ binding buffer at a concentration of $1 \times 10^{6}$ cells $/ \mathrm{ml}$. After this, $5 \mu \mathrm{L}$ fluorescein isothiocynate (FITC)-conjugated Annexin-V and $10 \mu \mathrm{L}$ PI were added to each tube and incubated for 15 min at room temperature. Then, $400 \mu \mathrm{L} 1 \times$ binding buffer was added to each tube before acquisition and analysis using a flow cytometer.

\section{Real-time quantitative PCR (RT-qPCR)}

Total RNA was extracted using TRIZOL (Invitrogen Life Technologies, Carlsbad, CA, USA). RNA (2.5 $\mu \mathrm{g}$ ) was reverse-transcribed using the Superscript ${ }^{\mathrm{TM}}$ III kit (Invitrogen) according to the manufacturer's instructions. The genes were amplified from the cDNA by PCR. The cDNA was amplified by PCR using the specific primers. The PCR was performed using an initial step of denaturation $\left(5 \mathrm{~min}\right.$ at $\left.94^{\circ} \mathrm{C}\right), 20-28$ cycles of amplification $\left(94^{\circ} \mathrm{C}\right.$ for $30 \mathrm{~s}, 54-58^{\circ} \mathrm{C}$ for $1 \mathrm{~min}$ and $72^{\circ} \mathrm{C}$ for $\left.1 \mathrm{~min}\right)$ and an extension $\left(72^{\circ} \mathrm{C}\right.$ for $\left.5 \mathrm{~min}\right)$. RTqPCR was performed by using One Step SYBR $®$ PrimeScript $®$ PLUS RT-RNA PCR Kit (TaKaRa Biotechnology, Dalian, China). The GAPDH served as the internal control for sample loading and mRNA integrity. The data was analyzed by the $2^{-\Delta \Delta t}$ method [21].

\section{Western Blot Assay}

The proteins from these cells werelysed using RIPA buffer ( $1 \times$ PBS, $1 \%$ NP- $40,0.5 \%$ sodium deoxycholate and $0.1 \%$ SDS, containing fresh protease inhibitor cocktail, Beyotime Biotechnology, Shanghai, China). The electrophoresis and immunoblotting procedures were performed according to a previous report [22]. The membranes were probed with primary antibodies of anti-Bcl-2 (ab32124), anti-Bax (ab53154), anti-Ecadherin (ab15148), anti-N-cadherin (ab18203), anti-Vimentin (ab16700), anti- $\beta$-catenin (ab32572), antip-ERK1/2 (ab214362), anti-ERK1/2 (ab17942), anti-p-PI3K (ab182651), anti-PI3K (ab40755), anti-p-AKT (ab38449), anti-AKT (ab18785), anti-Ki-67 (ab15580), anti-MMP-2 (ab37150), and GAPDH (ab181602). 
After incubation with the appropriate primary antibodies, the membranes were incubated for $1 \mathrm{~h}$ at room temperature with a secondary antibody conjugated to horseradish peroxidase (HRP)-conjugated goat antirabbit IgG (ab205718, 1:2000, Abcam). The blots were visualized by a PowerOpti-ECL (Animal Genetics Inc, Tallahassee, FL, USA) detection system according to the recommended procedure.

\section{Tumor Xenograft Model In vivo}

Thirty two male BALB/c nude mice of 5 weeks old were obtained from the Experimental Animal Center of Jilin University (Changchun, China), and these mice were treated according to the local ethics guidelines for animal research. Subsequently, MKN-45 cells in $0.1 \mathrm{ml}$ of serum-free RPMI-1640 were injected subcutaneously with a 24-gauge needle into the right flank of each nude mouse. The mice were randomized for therapy in four experimental groups, and $200 \mu \mathrm{g}$ of rat $\alpha$-mouse PD-1 (29F.1A12) or 100 $\mu \mathrm{g}$ of $\alpha$-CTLA-4 (clone 9D9) were administered intraperitoneally (i.p.) 3, 6, 9 and 12 days (every 3 days for a total of four times). Rat IgG was used as a control group. The tumor volume was detected according to previous study [23]. All studies involving animals were approved by the Ethics Committee of our institution.

\section{Statistical analysis}

Statistical differences between groups were analyzed by Student's $t$-test and one-way analysis of variance (ANOVA) for parametric data. Data are expressed as mean \pm standard deviation (SD). $\mathrm{P}<0.05$ was set as the level of statistical significance.

\section{Results}

Combination of anti-CTLA-4 and anti-PD-1 antibodies suppressed cell proliferation

To explore the effect of anti-CTLA- 4 and anti-PD-1 on cell proliferation, CCK-8 assay was performed to examine the viability of MKN-45 and MGC-803. As shown in Fig. $1 \mathrm{~A}$ and $1 \mathrm{~B}$, cell viability was significantly suppressed in all the three test groups of CTLA-4, PD-1, and CTLA-4\&PD-1 compared to control group in both MKN-45 and MGC803 cell lines. However, suppression of cell viability was higher in the group receiving both anti-CTLA-4 and anti-PD- 1 antibodies $(\mathrm{P}<0.01)$ than in anti-CTLA-4 $(\mathrm{P}<0.05)$ at $24 \mathrm{~h}(89.7 \%$ or $89.1 \%), 48 \mathrm{~h}(88.6 \%$ or $88.3 \%)$, and $72 \mathrm{~h}(88.5 \%$ or $88.4 \%)$, antiPD-1 $(\mathrm{P}<0.05)$ at $24 \mathrm{~h}(90.9 \%$ or $90.4 \%)$, $48 \mathrm{~h}(88.6 \%$ or $88.3 \%)$, and $72 \mathrm{~h}(85.3 \%$ or $85.1 \%)$, and control group $(\mathrm{P}<0.01)$ at $24 \mathrm{~h}$ (74.5\% or $73.1 \%), 48 \mathrm{~h}(68.6 \%$ or $67.8 \%)$, and $72 \mathrm{~h}(66.2 \%$ or $65.8 \%)$ in MKN-45 cells or MGC-803 cells. To further investigate whether these conditions influence the different phases of cell division, cell cycle was then examined by flow cytometry. The

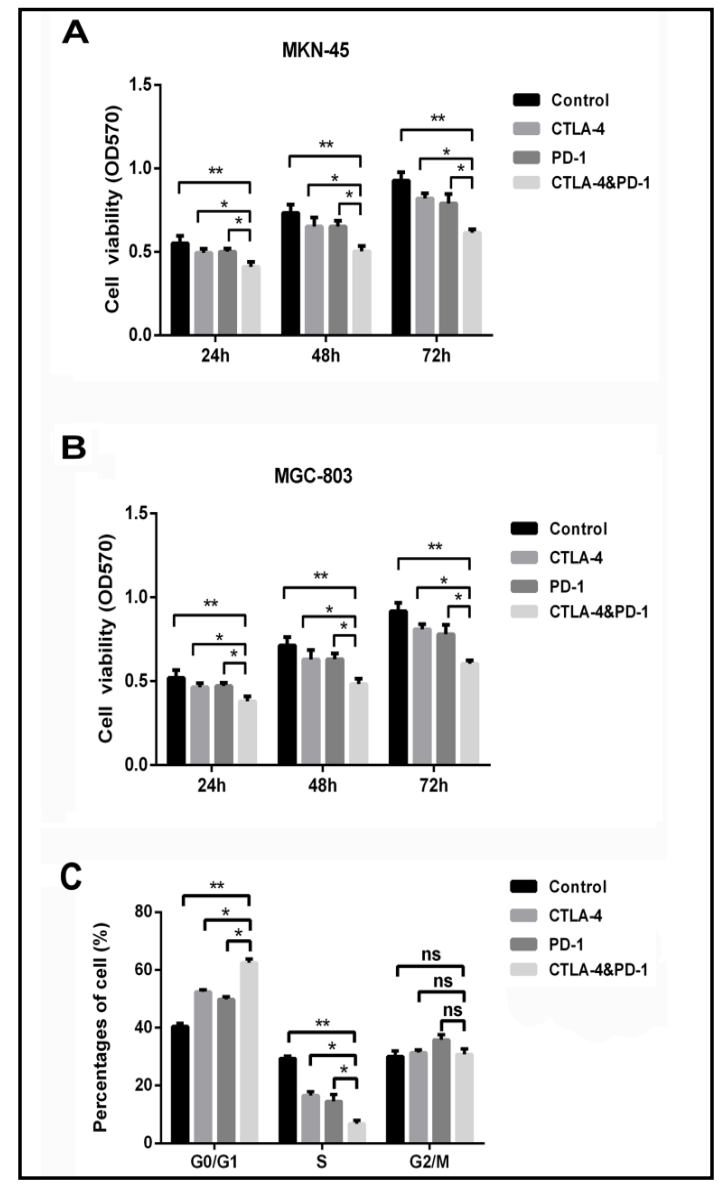

Fig. 1. Combination of anti-CTLA-4 and anti-PD-1 antibodies suppressed cell proliferation in MKN45 cells or MGC-803 cells. A: Combination of antiCTLA-4 and anti-PD-1 antibodies suppressed cell proliferation in $\mathrm{MKN}-45$ cell line; B: Combination of anti-CTLA-4 and anti-PD-1 antibodies suppressed cell proliferation in MGC-803 cell line; C: Combination of anti-CTLA-4 and anti-PD-1 antibodies arrested MKN-45 cells at G0/G1 phase. CTLA-4: cytotoxic T-lymphocyte-associated protein 4; PD-1: Programmed cell death protein $1 ;{ }^{*} \mathrm{P}<0.05$, ${ }^{* *} \mathrm{P}<0.01$, ns: non significance. 
results in Fig. 1C showed that the cell percentage at G0/G1 phase significantly increased in anti-CTLA-4 and anti-PD- 1 treated cells compared with control group $(\mathrm{P}<0.05)$. These data suggested that combination of anti-CTLA- 4 and anti-PD- 1 antibodies could inhibit cell proliferation in GC cells.

\section{Combination of anti-CTLA-4 and anti-PD-1 antibodies promoted cell apoptosis}

Flow cytometry analysis of cell apoptosis revealed that the percentage of apoptotic cells was significantly increased in MKN and MGC-803 cells treated with both anti-CTLA-4 and anti-PD- 1 antibodies compared to control group ( $\mathrm{P}<0.05$ or $\mathrm{P}<0.01$, Fig. $2 \mathrm{~A}$ and $2 \mathrm{~B}$ ). RT-qPCR assay revealed that the mRNA expression level of Bcl-2 was significantly decreased in cells treated with both anti-CTLA-4 and anti-PD-1 antibodies compared to in control group cells $(\mathrm{P}<0.05$ or $\mathrm{P}<0.01)$, as well as the expression level of Bax was significantly increased in MKN and MGC-803 cells treated with both anti-CTLA-4 and anti-PD-1 antibodies compared to the control group $(\mathrm{P}<0.05$ or $\mathrm{P}<0.01$, Fig. 2C). These results were further confirmed by western blot analysis, which demonstrated the suppression of $\mathrm{Bcl}-2$ expression and promotion of Bax expression in cells treated with both antiCTLA-4 and anti-PD-1 antibodies (Fig. 2D). These data indicated that combination of anti-CTLA-4 and anti-PD-1 antibodies could induce cell apoptosis.

Combination of antiCTLA-4 and anti-PD-1 antibodies inhibited cell migration, invasion and EMT

Next, transwell assay was performed to determine the migration and invasiveness of MKN and MGC-803 cells. The results demonstrated that combined administration of anti-CTLA-4 and anti-PD-1 antibodies significantly suppressed cell migration and invasion in both MKN-45 and MGC-803 cells compared to control group $(\mathrm{P}<0.01)$, only antiCTLA-4 antibody treated group $(\mathrm{P}<0.05)$, and only anti-PD-1 antibody treated

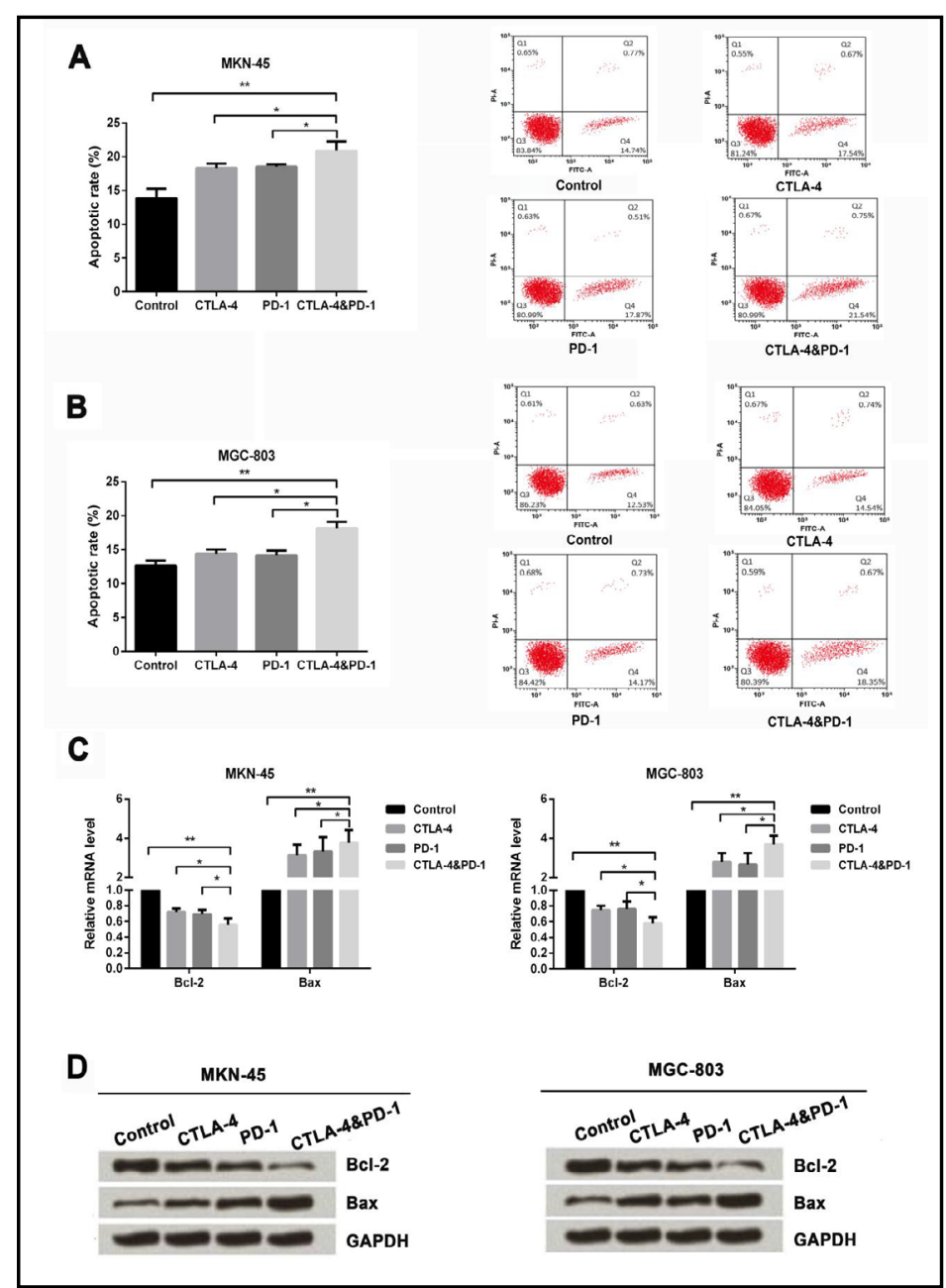

Fig. 2. Combination of anti-CTLA- 4 and anti-PD-1 antibodies induced cell apoptosis in MKN-45 cells or MGC-803 cells. A and B: Combination of anti-CTLA- 4 and anti-PD- 1 antibodies promoted apoptosis in MKN45 cells and MGC-803 cells; C: Combination of anti-CTLA- 4 and antiPD-1 antibodies regulated the mRNA and protein levels of apoptosis related factors in MKN- 45 cells and MGC-803 cells by using RT-qPCR and western blot analysis. CTLA-4: cytotoxic T-lymphocyte-associated protein 4; PD-1: Programmed cell death protein 1; RT-qPCR: real-time quantitative PCR; ${ }^{*} \mathrm{P}<0.05,{ }^{* *} \mathrm{P}<0.01$. 
Fig. 3. Combination of anti-CTLA-4 and anti-PD-1 antibodies inhibited cell migration and invasion in MKN-45 cells or MGC-803 cells. A: Combination of anti-CTLA-4 and anti-PD-1 antibodies inhibited cell migration and invasion in $\mathrm{MKN}-$ 45 cells; B: Combination of antiCTLA-4 and anti-PD-1 antibodies inhibited cell migration and invasion in MGC-803 cells.CTLA-4: cytotoxic T-lymphocyte-associated protein 4; PD-1: Programmed cell death protein $1 ;{ }^{*} \mathrm{P}<0.05$, ${ }^{* *} \mathrm{P}<0.01$.
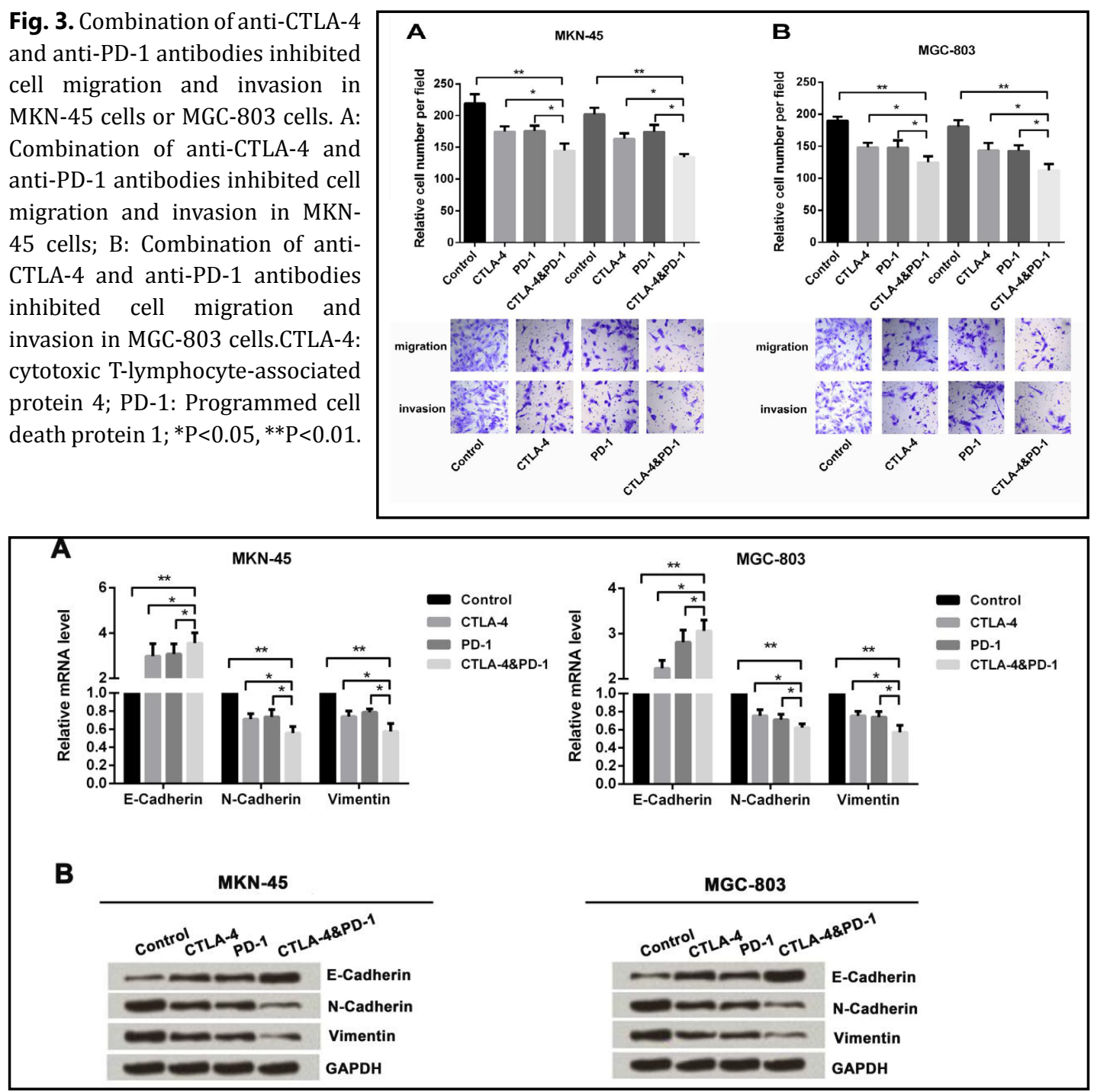

Fig. 4. Combination of anti-CTLA-4 and anti-PD-1 antibodies inhibited EMT in MKN-45 cells or MGC803 cells. A: Combination of anti-CTLA-4 and anti-PD-1 antibodies inhibited the mRNA expression levels of EMT-associated factors in MKN-45 cells and MGC-803 cells by using RT-qPCR; B: Combination of antiCTLA-4 and anti-PD-1 antibodies inhibited the protein levels of EMT-associated factors in MKN-45 cells and MGC-803 cells by using western blot analysis. CTLA-4: cytotoxic T-lymphocyte-associated protein 4; PD-1: Programmed cell death protein 1; RT-qPCR: real-time quantitative PCR; EMT: epithelial mesenchymal transition; ${ }^{*} \mathrm{P}<0.05,{ }^{*} \mathrm{P}<0.01$.

group $(\mathrm{P}<0.05$, Fig. $3 \mathrm{~A}$ and $3 \mathrm{~B})$. RT-qPCR was performed to measure the related factors of EMT process. It was found that combination of anti-CTLA-4 and anti-PD-1 antibodies significantly suppressed EMT as evidenced by increasing E-cadherin expression, and suppressing $\mathrm{N}$-cadherin and Vimentin expression compared to control group ( $\mathrm{p}<0.01)$, only anti-CTLA- 4 antibody treated group $(\mathrm{P}<0.05)$, and anti-PD- 1 antibody treated group $(\mathrm{P}<0.05$, Fig. 4A). Furthermore, western blot analysis supported these findings, as the expression of E-cadherin protein was maximum while the expression of $\mathrm{N}$-cadherin and Vimentin were minimum in cells treated with both anti-CTLA- 4 and anti-PD- 1 antibodies compared to other groups (Fig. 4B). All above results displayed that combination of anti-CTLA-4 and anti-PD-1 antibodies could inhibit cell migration, invasion and EMT in GC cells. 


\section{Cellular Physiology Cell Physiol Biochem 2018;49:260-270 and Biochemistry \begin{tabular}{l|l} 
DOI: 10.1159/000492876 & (c) 2018 The Author(s). Published by S. Karger AG, Basel \\
www.kargercom/cpb
\end{tabular}

Combination of anti-CTLA-4 and anti$P D-1$ antibodies suppressed the activation of $\beta$-catenin, MAPK and PI3K/AKT signal pathways

Next, the protein levels of $\beta$-catenin pathwayassociated factors were explored in different groups by using western blot analysis. It was found that the protein levels of total $\beta$-catenin, Nucleus and Cytoplasm were notably decreased in the cells treated with both anti-CTLA- 4 and anti-PD-1 antibodies compared to other groups of cells (Fig. 5A and 5B). Additionally, we found that both anti-CTLA-4 and anti-PD-1 antibodies suppressed the protein levels of phosphorylated ERK1/2, PI3K and AKT compared to other groups of cells (Fig. 6). There was no significant change of ERK1/2, PI3K and AKT in different groups. These data indicated that combination of anti-CTLA-4 and anti-PD-1 antibodies could block $\beta$-catenin, MAPK and PI3K/AKT signal pathways, thereby affecting cell proliferation, apoptosis, migration and invasion in GC cells.

Combination of anti-CTLA-4 and anti-PD-1 antibodies inhibited tumor formation in vivo

Finally, the effect of anti-CTLA-4 and antiPD-1 antibodies on tumor formation in vivo was investigated. As shown in Fig. 7, the results displayed that combination of anti-CTLA-4 and anti-PD-1 antibodies significantly inhibited tumor volume compared with control group $(\mathrm{p}<0.001)$, only anti-CTLA-4 antibody treated group $(\mathrm{P}<0.01)$, and only anti-PD-1 antibody treated group $(\mathrm{P}<0.05)$ after treatment for 6,9 and 12 days, respectively. These data indicated that anti-CTLA-4 and anti-PD-1 antibodies could suppress tumor formation in vivo.

Combination of CTLA-4 silencing and $P D-1$ silencing regulated cell proliferation, apoptosis and metastasis in MKN45 cells

To further investigate the role of CTLA-4 and $P D-1$ in GC cells proliferation, apoptosis, migration, invasion and EMT was examined. The plasmids of si-CTLA- 4 and si-PD-1 were transfected into MKN45 cells, and RT-qPCR assay showed that the mRNA levels of CTLA-4 and PD-1 were significantly down-regulated by CTLA-4 silencing or PD-1 silencing compared with siNC group $(\mathrm{P}<0.01$, Fig. $8 \mathrm{~A})$. The combination of CTLA-4 silencing and PD-1 silencing significantly suppressed cell viability, induced apoptosis, as well as down-regulated $\mathrm{Bcl}-2$ and up-regulated Bax expression in MKN45 cells $(\mathrm{P}<0.05$ or

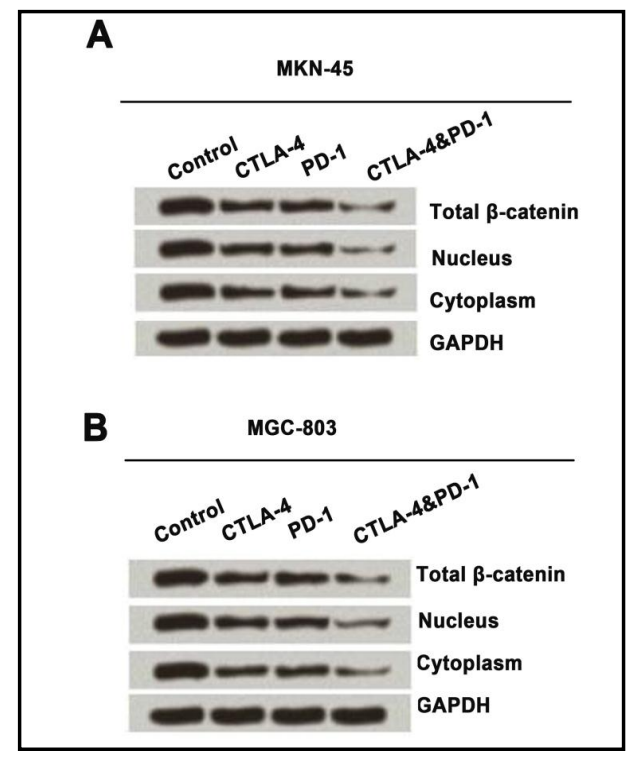

Fig. 5. Combination of anti-CTLA-4 and antiPD-1 antibodies blocked $\beta$-catenin signal pathway in MKN-45 cells or MGC-803 cells. A: Combination of anti-CTLA-4 and antiPD-1 antibodies suppressed the protein levels total $\beta$-catenin; nucleus and Cytoplasm in MKN-45 cells; (B) Combination of antiCTLA-4 and anti-PD-1 antibodies suppressed the protein levels total $\beta$-catenin; nucleus and Cytoplasm in MGC-803 cells. CTLA-4: cytotoxic T-lymphocyte-associated protein 4; PD-1: Programmed cell death protein 1.

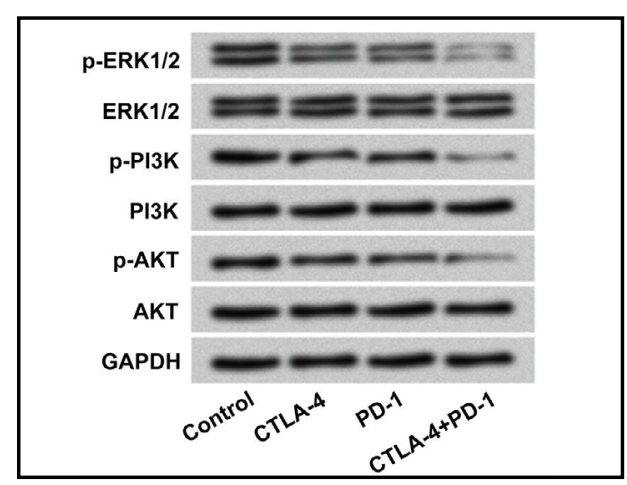

Fig. 6. Combination of anti-CTLA-4 and antiPD-1 antibodies blocked MAPK and PI3K/AKT signal pathways in MKN-45 cells. The protein levels of phosphorylated ERK1/2, PI3K and AKT were declined by combination of antiCTLA-4 and anti-PD-1 antibodies in MKN45 cells. CTLA-4: cytotoxic T-lymphocyteassociated protein 4; PD-1: Programmed cell death protein 1; MAPK: mitogen-activated protein kinase; PI3K: phosphatidylinositol-3 kinase; AKT: protein kinase B. 
Fig. 7. Combination of anti-CTLA-4 and anti-PD-1 antibodies inhibited tumor formation in vivo. The tumor volume was significantly inhibited by combination of anti-CTLA-4 and anti-PD-1 antibodies in vivo after treatment for 6, 9 and 12 days, respectively. ${ }^{*} \mathrm{P}<0.05$ compared to CTLA4 antibody group; \#P<0.05 compared to $\mathrm{PD}-1$ antibody group; $\& \mathrm{P}<0.05, \& \& \mathrm{P}<0.01, \& \& \& \mathrm{P}<0.001$ compared to IgG group.

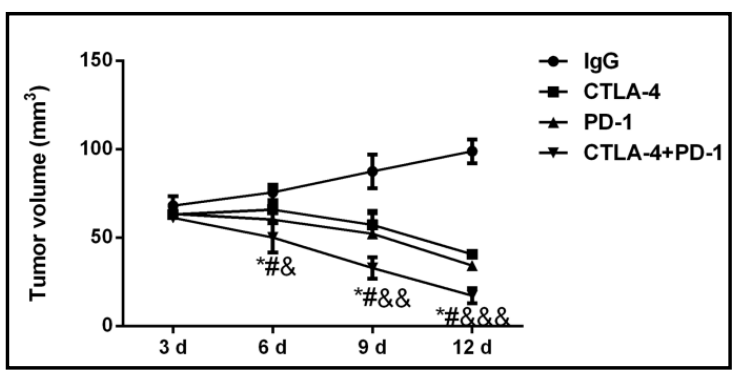

A

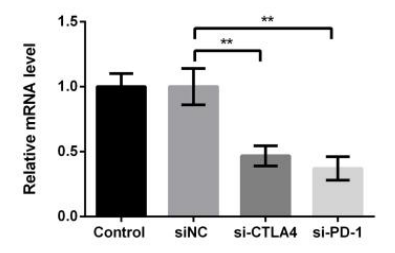

C

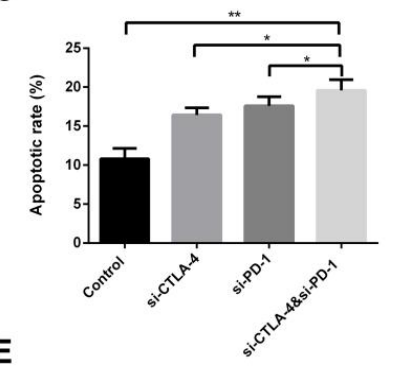

E

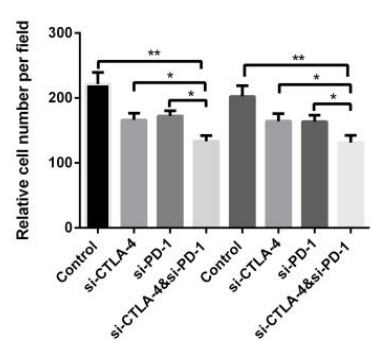

B

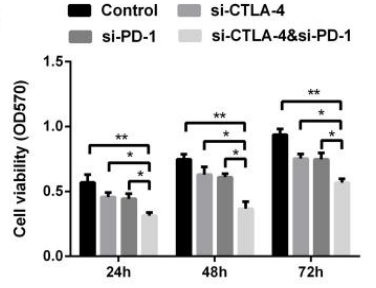

D
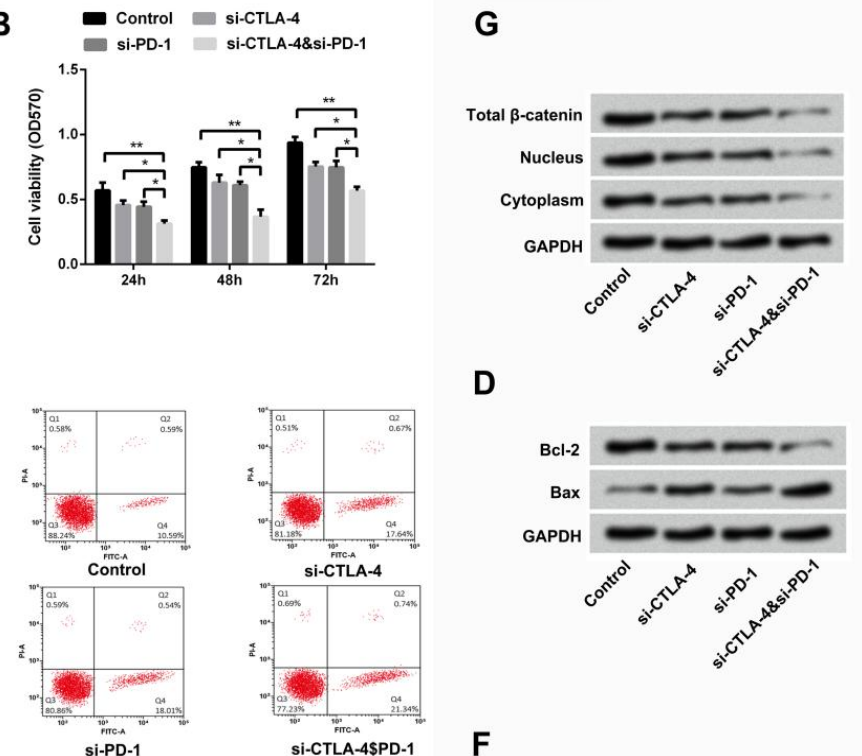

Si-CTLA-4SPD-1

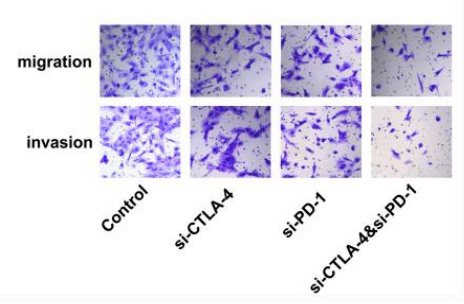

Fig. 8. Combination of CTLA-4 silencing and PD-1 silencing regulated cell proliferation, apoptosis and metastasis in MKN45 cells. A: MKN45 cells were transfected with si-CTLA-4 and si-PD-1, and the mRNA expression levels of CTLA-4 and PD-1 were significantly decreased in these transfected cells; combination of CTLA-4 silencing and PD-1 silencing B: suppressed cell viability, C: induced apoptosis, D: down-regulated Bcl-2 and up-regulated Bax expression, E: inhibited migration and invasion, F: suppressed EMT-associated factors, as well as G: inactivated $\beta$-catenin signal pathway in MKN-45 cells. CTLA-4: cytotoxic T-lymphocyteassociated protein 4; PD-1: Programmed cell death protein 1; EMT: epithelial mesenchymal transition; ${ }^{*} \mathrm{P}<0.05,{ }^{* *} \mathrm{P}<0.01$.

$\mathrm{P}<0.01$, Fig. 8B-8D). Moreover, cell migration and invasion were also decreased by CTLA4 silencing and PD- 1 silencing $(\mathrm{P}<0.05$, Fig. 8E). Meanwhile, CTLA-4 silencing and PD-1 silencing increased the protein level of E-cadherin, and decreased the protein levels of $\mathrm{N}$-cadherin and Vimentin in MKN45 cells (Fig. 8F). Further, we found that the protein levels of total $\beta$-catenin, Nucleus and Cytoplasm were down-regulated by CTLA- 4 silencing and PD-1 silencing in MKN45 cells (Fig. 8G). All these data indicated that combination of CTLA-4 silencing and PD-1 silencing could inhibit cell proliferation, metastasis and induce apoptosis in MKN45 cells. 


\section{Discussion}

Approximately two-thirds of the patients with GC have been unable to receive the tumor resection treatment or presented the distant organs metastasis at the initial diagnosis. Even with screening programs, more than $80 \%$ GC patients also happened locally or distally recurrence after surgery [24]. Current understanding of GC pathophysiology from biological and genomic perspective has led to the development of target-oriented therapy [24, 25]. Immunotherapy has been proven to play a major role in target-oriented therapy $[7,10,14$, 19]. Both CTLA-4 and PD-1 antibodies are widely utilized in immunotherapy in recent years. Several studies have already explored the role of anti-CTLA-4 and anti-PD-1 antibodies in the management of various cancers, including GC $[19,26]$. In the present study, we have explored the combined role of anti-CTLA-4 and anti-PD-1 antibodies in GC cells. Results showed that combination of anti-CTLA-4 and anti-PD-1 antibodies significantly suppressed cell viability, migration, invasion and promoted apoptosis in both MKN and MGC-803 cells. Additionally, we also demonstrated that combination of anti-CTLA- 4 and anti-PD- 1 antibodies inhibited EMT process, as increasing E-cadherin expression and decreasing Vimentin and N-cadherin expression. Further, treatment with anti-CTLA- 4 and anti-PD- 1 antibodies blocked $\beta$-catenin, MAPK and PI3K/AKT pathways in GC cells. Finally, we found that combination of anti-CTLA-4 and anti-PD-1 antibodies inhibited tumor formation in vivo.

Several studies have proven the anticancer effect of anti-CTLA- 4 antibody on different types of cancers, such as non-small cell lung cancer (NSCLC), melanoma, and GC [15, 19, 26-29]. Ralph et al. conducted the clinical trial with tremelimumab (a fully humanized anti-CTLA-4 monoclonal antibody) in eighteen patients with metastatic GC and esophageal adenocarcinoma, and the study revealed the promising role of CTLA-4 blockade along with other forms of immunotherapy [15]. Currently, anti-PD-1 antibody is approved for the management of untreated malignant melanoma and NSCLC. Moreover, several studies have explored its role in the management of other cancers, including ovarian cancer, prostate cancer, bladder cancer, and GC [30,31]. Evidence from Liu et al. demonstrated that antiPD-1 antibody might be a therapeutic approach for GC [4]. Based on these previous studies, we explored the effect of combination of anti-CTLA-4 and anti-PD-1 antibodies on GC cells. The results showed that combination of anti-CTLA-4 and anti-PD-1 antibodies significantly suppressed cell proliferation, migration, invasion, EMT, as well as induced apoptosis in GC cells. These data suggested that combination therapy with anti-CTLA-4 and anti-PD-1 antibodies might suppress the development of GC.

Several studies have discussed the role of $\beta$-catenin pathway in different types of cancer including GC, and these studies demonstrated that activation of $\beta$-catenin pathway was implicated in the progression of GC $[32,33]$. MAPK and PI3K/AKT signal pathways are important regulators in various cancers, and which are associated with the occurrence and development of GC [34]. Evidence from Qu et al. reported that activation of PI3K/AKT and MAPK/ERK could promote GC cells proliferation [35]. Shah et al. found that CTLA-4 was a direct target of Wnt/ $\beta$-catenin signaling and was expressed in human melanoma tumors [36]. Further, Deken et al. demonstrated that targeting the MAPK and PI3K pathways in combination with PD-1 could blockade in melanoma [37]. However, whether $\beta$-catenin, MAPK and PI3K/AKT signal pathways could influence the anticancer effect of anti-CTLA-4 and anti-PD-1 antibodies on GC cells remains uninvestigated. These data indicated that anti-CTLA-4 and anti-PD-1 antibodies regulated cell proliferation, apoptosis and metastasis might through inactivation of $\beta$-catenin, MAPK and PI3K/AKT signal pathways. Finally, in vivo experiment was performed to further confirm the effect of anti-CTLA-4 and anti-PD-1 antibodies on tumor formation. The results revealed that combination of anti-CTLA-4 and anti-PD-1 antibodies inhibited tumor formation in vivo. Thus, these data suggested that combination therapy with anti-CTLA- 4 and anti-PD-1 antibodies have shown encouraging results in GC. 


\section{Cellular Physiology Cell Physiol Biochem 2018:49:260-270 \begin{tabular}{l|l|l}
\hline and BOI: 10.1159/000492876 & $\begin{array}{l}\text { C } 2018 \text { The Author(s). Published by S. Karger AG, Basel } \\
\text { www.karger.com/cpb }\end{array}$ \\
\hline
\end{tabular}}

Wang et al.: Effects of Anti-CTLA-4 and Anti-PD-1 on GC

Taken together, these data indicated that combination of anti-CTLA- 4 and anti-PD-1 antibodies could inhibit cell proliferation, migration, invasion, EMT, and induce apoptosis in GC cells through regulation of $\beta$-catenin, MAPK and PI3K/AKT signal pathways. These finding might provide a new sight for the treatment of GC, and widely effect of anti-CTLA-4 and anti-PD-1 antibodies on GC might warrant further investigation.

\section{Acknowledgements}

The work received no funding support.

\section{Disclosure Statement}

The authors declare to have no conflict of interests.

\section{References}

1 Matsueda S, Graham DY: Immunotherapy in gastric cancer. World J Gastroenterol 2014;20:1657-1666.

2 Vial M, Grande L, Pera M: Epidemiology of adenocarcinoma of the esophagus, gastric cardia, and upper gastric third. Recent Results Cancer Res 2010;182:1-17.

3 Jemal A, Siegel R, Xu J, Ward E: Cancer statistics, 2010 CA Cancer J Clin 2010;60:277-300.

$\checkmark 4$ Liu X, Yang Z, Latchoumanin O, Liang Q: Antagonizing programmed death-1 and programmed death ligand-1 as a therapeutic approach for gastric cancer. Therap Adv Gastroenterol 2016;9:853-860.

5 Joo MK, Park JJ, Chun HJ: Recent updates of precision therapy for gastric cancer: Towards optimal tailored management. World J Gastroenterol 2016;22:4638-4650.

$>6$ Ku GY, Ilson DH: Management of gastric cancer. Curr Opin Gastroenterol 2014;30:596-602.

Thrumurthy SG, Chaudry MA, Hochhauser D, Mughal M: The diagnosis and management of gastric cancer. BMJ 2013;347:f6367.

-8 Lee JH, Kim KM, Cheong JH, Noh SH: Current Management and Future Strategies of Gastric Cancer. Yonsei Med J 2012;53:248-257.

9 Niccolai E, Taddei A, Prisco D, Amedei A: Gastric cancer and the epoch of immunotherapy approaches. World J Gastroenterol 2015;21:5778-5793.

10 Subhash VV, Mei SY, Tan WL, Yong WP: Strategies and Advancements in Harnessing the Immune System for Gastric Cancer Immunotherapy. J Immunol Res 2014;2015:1-14.

11 Yu J, Wu J, Liu S, Zhou G, Sanchez R, Dawson D, Nemunaitis J, Brunicardi FC: Immune Response Plays an Important Role in KRASG12D Induced Tumorigenesis in PDX1 Expressing Cells in Pancreas. J Surg Res 2014;186:502-502.

12 Goode EF, Smyth EC: Immunotherapy for Gastroesophageal Cancer. J Clin Med 2016;5:84.

$>13$ Chesler L: Paraneoplasia, cancer development and immunity: what are the connections? Nat Rev Cancer 2014;14:447-448.

$>14$ Chen DS, Mellman I: Oncology meets immunology: the cancer-immunity cycle. Immunity 2013;39:1-10.

15 Ralph C, Elkord E, Burt DJ, O’Dwyer JF, Austin EB, Stern PL, Hawkins RE, Thistlethwaite FC: Modulation of lymphocyte regulation for cancer therapy: a phase II trial of tremelimumab in advanced gastric and esophageal adenocarcinoma. Clin Cancer Res 2010;16:1662-1672.

$>16$ Okita R, Yamaguchi Y, Emi A, Matsuura K, Toge T: Enhancement of lymphokine-activated killer cell induction using anti-CD25 and anti-CTLA-4 monoclonal antibodies. Oncol Rep 2007;17:1429-1435.

17 Savabkar S, Azimzadeh P, Chaleshi V, Nazemalhosseini ME, Aghdaei HA: Programmed death-1 gene polymorphism (PD-1.5 C/T) is associated with gastric cancer. Gastroenterol Hepatol Bed Bench 2013;6:178-182.

18 Ma C, Patel K, Singhi AD, Ren B, Zhu B, Shaikh F, Sun W: Programmed Death-Ligand 1 Expression Is Common in Gastric Cancer Associated With Epstein-Barr Virus or Microsatellite Instability. Am J Surg Pathol 2016;40:1496-1506. 


\section{Cellular Physiology Cell Physiol Biochem 2018;49:260-270 \begin{tabular}{ll|l} 
DOI: 10.1159/000492876 & $\begin{array}{l}\text { O } 2018 \text { The Author(s). Published by S. Karger AG, Basel } \\
\text { www.karger.com/cpb }\end{array}$
\end{tabular}}

Wang et al.: Effects of Anti-CTLA-4 and Anti-PD-1 on GC

19 Cetin B, Gumusay 0, Cengiz M, Ozet A: Advances of Molecular Targeted Therapy in Gastric Cancer. J Gastrointest Cancer 2016;47:125-134.

20 Lote H, Cafferkey C, Chau I: PD-1 and PD-L1 blockade in gastrointestinal malignancies. Cancer Treat Rev 2015;41:893-903.

-21 Livak KJ, Schmittgen TD: Analysis of relative gene expression data using real-time quantitative PCR and the 2(-Delta Delta C(T)) Method. Methods 2001;25:402-408.

-22 Zhou J, Liu Z, Yu J, Han X, Fan S, Shao W, Chen J, Qiao R, Xie P: Quantitative Proteomic Analysis Reveals Molecular Adaptations in the Hippocampal Synaptic Active Zone of Chronic Mild Stress-Unsusceptible Rats. Int J Neuropsychoph 2015;19pii:pyv100.

-23 Duraiswamy J, Freeman GJ, Coukos G: Dual Blockade of PD-1 and CTLA-4 Combined with Tumor Vaccine Effectively Restores T-Cell Rejection Function in Tumors-Response. Cancer Res 2013;73:633-634.

24 Sun W, Yan L: Gastric cancer: current and evolving treatment landscape. Chin J Cancer 2016;35:83.

25 Liu W, Zhang X, Sun W: Developments in treatment of esophageal/gastric cancer. Curr Treat Options Oncol 2008;9:375-387.

26 Peggs KS, Quezada SA, Korman AJ, Allison JP: Principles and use of anti-CTLA4 antibody in human cancer immunotherapy. Curr Opin Immunol 2006;18:206.

27 Xia B, Herbst RS: Immune checkpoint therapy for non-small-cell lung cancer: an update. Immunotherapy 2016;8:279-298.

28 Kwek SS, Cha E, Fong L: Unmasking the immune recognition of prostate cancer with CTLA4 blockade. Nat Rev Cancer 2011;12:289-297.

29 Lens M, Ferrucci PF, Testori A: Anti-CTLA4 monoclonal antibody Ipilimumab in the treatment of metastatic melanoma: recent findings. Recent Pat Anticancer Drug Discov 2008;3:105-113.

-30 Hatae R, Chamoto K: Immune checkpoint inhibitors targeting programmed cell death-1 (PD-1) in cancer therapy. Rinsho ketsueki 2016;57:2224-2231.

-31 Swaika A, Hammond WA, Joseph RW: Current state of anti-PD-L1 and anti-PD-1 agents in cancer therapy. Mol Immunol 2015;67:4-17.

32 Jamieson C, Sharma M, Henderson BR: Targeting the $\beta$-catenin nuclear transport pathway in cancer. Semin Cancer Biol 2014;27:20-29.

33 Li-Fu, Zheng-Jie, Hong, Jiang: Abnormal $\beta$-catenin immunohistochemical expression as a prognostic factor in gastric cancer: A meta-analysis. World J Gastroenterol 2014;20:12313-12321.

34 Akrami H, Mahmoodi F, Havasi S, Sharifi A: PlGF knockdown inhibited tumor survival and migration in gastric cancer cell via PI3K/Akt and p38MAPK pathways. Cell Biochem Funct 2016;34:173-180.

-35 Qu JL, Qu XJ, Zhao MF, Teng YE, Zhang Y, Hou KZ, Jiang YH, Yang XH, Liu YP: Gastric cancer exosomes promote tumour cell proliferation through PI3K/Akt and MAPK/ERK activation. Dig Liver Dis 2009;41:875-880.

-36 Shah KV, Chien AJ, Yee C, Moon RT: CTLA-4 is a direct target of Wnt/beta-catenin signaling and is expressed in human melanoma tumors. J Invest Dermatol 2008;128:2870-2879.

-37 Deken MA, Gadiot J, Jordanova ES, Lacroix R, van Gool M, Kroon P, Pineda C, Geukes Foppen MH, Scolyer R, Song JY, Verbrugge I, Hoeller C, Dummer R, Haanen JBAG, Long GV, Blank CU: Targeting the MAPK and PI3K pathways in combination with PD1 blockade in melanoma. Oncoimmunology 2016;5:e1238557. 